\title{
Precision measurements of the charge radii of potassium isotopes
}

\author{
Á. Koszorús, ${ }^{1}$ X. F. Yang, ${ }^{2,1,}{ }^{*}$ J. Billowes,${ }^{3}$ C. L. Binnersley ${ }^{3}$ M. L. Bissell, ${ }^{3}$ T. E. Cocolios, ${ }^{1}$ G. J. Farooq-Smith,${ }^{1}$ \\ R. P. de Groote, ${ }^{1,4}$ K. T. Flanagan, ${ }^{3,5}$ S. Franchoo, ${ }^{6}$ R. F. Garcia Ruiz, ${ }^{3,7}$ S. Geldhof, ${ }^{4}$ W. Gins,,${ }^{1,4}$ A. Kanellakopoulos, ${ }^{1}$ \\ K. M. Lynch, ${ }^{7}$ G. Neyens, ${ }^{1,7}$ H. H. Stroke, ${ }^{8}$ A. R. Vernon, ${ }^{3,1}$ K. D. A. Wendt, ${ }^{9}$ and S. G. Wilkins ${ }^{3,10}$ \\ ${ }^{1}$ KU Leuven, Instituut voor Kern- en Stralingsfysica, B-3001 Leuven, Belgium \\ ${ }^{2}$ School of Physics and State Key Laboratory of Nuclear Physics and Technology, Peking University, Beijing 100871, China \\ ${ }^{3}$ School of Physics and Astronomy, The University of Manchester, Manchester M13 9PL, United Kingdom \\ ${ }^{4}$ Department of Physics, University of Jyväskylä, PB 35(YFL) FIN-40351 Jyväskylä, Finland \\ ${ }^{5}$ Photon Science Institute Alan Turing Building, University of Manchester, Manchester M13 9PY, United Kingdom \\ ${ }^{6}$ Institut de Physique Nucléaire Orsay, IN2P3/CNRS, 91405 Orsay Cedex, France \\ ${ }^{7}$ Physics Department, CERN, CH-1211 Geneva 23, Switzerland \\ ${ }^{8}$ Department of Physics, New York University, New York, New York 10003, USA \\ ${ }^{9}$ Institut für Physik, Johannes Gutenberg-Universität, D-55128 Mainz, Germany \\ ${ }^{10}$ Engineering Department, CERN, CH-1211 Geneva 23, Switzerland
}

(Received 5 March 2019; published 9 September 2019)

\begin{abstract}
Precision nuclear charge radii measurements in the light-mass region are essential for understanding the evolution of nuclear structure, but their measurement represents a great challenge for experimental techniques. At the Collinear Resonance Ionization Spectroscopy (CRIS) setup at ISOLDE-CERN, a laser frequency calibration and monitoring system was installed and commissioned through the hyperfine spectra measurement of ${ }^{38-47} \mathrm{~K}$. It allowed for the extraction of the hyperfine parameters and isotope shifts with better than $1 \mathrm{MHz}$ precision. These results are in excellent agreement with available literature values and they demonstrate the suitability of the CRIS technique for the study of nuclear observables in light atomic systems. In addition, the spectral line shapes obtained under different conditions were systematically investigated, highlighting the importance of finding optimal conditions, under which the extracted nuclear properties remain unaffected by laser-atom interactions.
\end{abstract}

DOI: 10.1103/PhysRevC.100.034304

\section{INTRODUCTION}

Precision laser spectroscopy can simultaneously measure multiple nuclear properties of isotopes in a nuclear modelindependent way, such as nuclear spins, electromagnetic moments, and changes in the mean-square charge radii. This makes such measurements a major ingredient for nuclear structure studies in different regions of the nuclear chart, providing important benchmarks for testing and developing state-of-the-art nuclear theories [1-8].

Over the years, various unexpected phenomena have been discovered in isotopes far from $\beta$ stability, challenging the well-established theoretical models initially based on the isotopes near the valley of stability. This has motivated continuous development of nuclear models as well as experimental techniques. As one of the fundamental properties of the atomic nucleus, the charge radii of short-lived isotopes

*xiaofei.yang@pku.edu.cn

Published by the American Physical Society under the terms of the Creative Commons Attribution 4.0 International license. Further distribution of this work must maintain attribution to the author(s) and the published article's title, journal citation, and DOI. have helped to probe (sub)shell effects, shape staggering, and correlations [4,9-11]. Nevertheless, their experimental measurements with high precision, as well as the accurate theoretical description of the charge radii remain challenging. Recently, significant theoretical efforts have been invested in the calculation of not only the general trend but also the local variations (e.g., odd-even staggering) of charge radii across a long isotopic chain in different regions of the nuclear chart $[4,10,12,13]$. The experimental challenge comes from the low production yields of nuclei far from $\beta$ stability. In addition, the presence of large amounts of isobaric contaminants that are often produced along these isotopes, further limits the applicability of existing experimental techniques. Considerable efforts have thus been dedicated to new instrumentation and methods, with the hope to overcome the aforementioned difficulties. This will allow the studies of isotopes far away from stability and will thus extend our knowledge of both nuclear and atomic $[7,14]$ properties in these systems produced at radioactive ion beam facilities.

Several laser spectroscopy methods can be used to investigate the nuclear charge radii $[2,15,16]$. Two techniques are widely used for the study of radioactive isotopes: in-source (or in-gas cell/jet) resonance ionization spectroscopy $[7,8,10,17]$, and collinear laser spectroscopy [18-20]. The former relies upon multistep resonance laser ionization of atoms close 
to the production point. The high efficiency of resonance laser ionization in combination with ion detection methods, provide a very high sensitivity, allowing the measurement of isotopes produced at rates below 1 particle per second [7,10,21-23]. However, the spectral resolution achieved with this class of techniques is, in general, not sufficient for highprecision measurements of isotopes in the light and mediummass region. Due to Doppler and pressure broadening effects, the width of the spectral line increases by two orders of magnitude compared to the natural linewidth, exceeding $1 \mathrm{GHz}$, and is therefore larger than the hyperfine splitting of most isotopes in the calcium mass region and below. The newly developed in-gas-jet resonance ionization spectroscopy has reached $400 \mathrm{MHz}$ linewidth for the actinium isotopes in the heavy-mass region [8], which is an order of magnitude improvement compared to the in-source technique. It is, however, still not enough to resolve the hyperfine structure of light elements. Collinear laser spectroscopy, on the other hand, reduces these effects by two orders of magnitude, due to kinematic compression that occurs when accelerating the ion beam to between $30-60 \mathrm{keV}[18,20]$. When the ion beam is overlapped with laser light in an (anti)collinear geometry [1,2], resonance profiles with less than $100 \mathrm{MHz}$ width can be obtained, reaching the order of magnitude of the natural linewidth. Currently, there are several new methods under development that aim to combine the high-efficiency resonance laser ionization process with high resolution [8,24,25].

Collinear laser spectroscopy typically brings the atoms or ions into resonance with a frequency-fixed laser light by changing the beam energy, while Collinear Resonance Ionization Spectroscopy (CRIS) established at CERN-ISOLDE [26] relies on changing the laser frequency. This approach used at CRIS has reached sufficient precision to reliably extract the nuclear properties of heavy-mass francium and radium isotopes [24,27,28] and medium-mass copper and gallium isotopes $[6,29]$. However, further developments were required to study light isotopes, and in particular for the extraction of their nuclear charge radii. Measurements of these radii are required to probe (sub)shell effects $[4,9,11]$, shape staggering [30,31], and correlations [32,33].

The changes in mean-square charge radii can be extracted from observed isotopes shifts $(\delta v)$ using the following expression [34]

$$
\delta v^{A A^{\prime}}=K_{\mathrm{MS}} \frac{m_{A^{\prime}}-m_{A}}{m_{A^{\prime}}\left(m_{A}-m_{e}\right)}+F \delta\left\langle r^{2}\right\rangle^{A A^{\prime}},
$$

where $K_{\mathrm{MS}}$ and $F$ are the atomic mass-shift and field-shift factors, respectively, of a given electronic transition, $m_{e}$ is the electron mass and $m_{\mathrm{A}}$ and $m_{\mathrm{A}^{\prime}}$ are nuclear masses. For light elements, the field shift, which is the critical part for the determination of the nuclear charge radii, is $10^{3}$ smaller than the mass shift [3]. Such is the case for the $\mathrm{K}$ isotopes (see $\mathrm{Sec}$. III C). For $\mathrm{K}$ isotopes, an inaccuracy at the level of a few $\mathrm{MHz}$ in $\delta v$ will lead to a significant error (more than 10\%) on the extracted charge radii. Therefore, for the measurement of light isotopes with $Z \leqslant 50$, it is essential to investigate the precision of the CRIS technique. Furthermore, the interaction of atoms with high-power laser pulses, required for efficient resonant ionization spectroscopy, leads to line shape distor- tions and shifts of the atomic energy levels [35], which can potentially influence the extracted nuclear parameters.

In the mass regions studied so far using the CRIS technique, these unwanted consequences of the use of high-power pulsed lasers were either successfully removed [36], or had little influence on the extracted nuclear properties, when compared with available literature values [29]. However, in the mass region of potassium, these effects have to be carefully investigated and addressed. Note that resonance ionization spectroscopy has been used to study the $\mathrm{Li}(Z=3)$ isotopes [37], which was possible by decoupling the two-photon Dopplerfree excitation and the resonance ionization processes through the decay of the first excited state. Therefore, the hyperfine structure of the two-photon excited atomic energy level, that was used to extract the nuclear parameters of the studied isotopes, was not affected by the high-power laser used for the ionization step.

In the past, high-precision laser spectroscopy of ${ }^{38-47} \mathrm{~K}$ was performed on thermal atomic beams [38], details of the technique can be found in Ref. [39], while the isotope shift of ${ }^{37-38} \mathrm{~K}$ was studied in a magneto-optic trap [40] with high precision. More recently, hyperfine spectra of ${ }^{36-51} \mathrm{~K}$ isotopes were measured with several collinear laser spectroscopy techniques on accelerated beams $[11,41,42]$. The availability of independent measurements using different methods for a long chain of isotopes therefore provides a perfect test site to investigate the precision and accuracy that can be achieved with the laser frequency scanning approach used at CRIS. This will also establish the accuracy limit of similar scanning methods for high-resolution laser spectroscopy techniques in general. In addition, the role of laser-induced distortion of spectral line shapes in resonance ionization spectroscopy $[29,43]$ can be further investigated. Although the study of laser-atom interactions has already attracted some attention $[35,43]$, to our knowledge these effects and in particular their impact on the nuclear properties extracted from hyperfine structure (hfs) spectra have not yet been studied in detail.

This work presents measurements of the hfs spectra of $38,39,42,44,46,47 \mathrm{~K}$ using the CRIS method, which were performed with a newly installed laser frequency monitoring and stabilization system. It will demonstrate the capability of the current experimental setup for reliable extraction of nuclear parameters in light isotopes. In addition, different experimental conditions (laser power, laser pulse timing structure) have been investigated to gain a better understanding of their influence on the line shape and extracted hfs constants, isotope shifts, and the deduced nuclear properties.

\section{EXPERIMENTAL METHOD AND SETUP}

The $\mathrm{K}$ isotopes were produced at the ISOLDE facility at CERN by impinging protons of $1.4 \mathrm{GeV}$ on a $20 \mathrm{~cm}$ long cylindrical $\mathrm{UC}_{\mathrm{x}}$ target $\left(45 \mathrm{~g} / \mathrm{cm}^{2}\right)$ with $0.7 \mathrm{~cm}$ diameter [44]. Reaction products diffused out of the target into a capillary tube (3 mm diameter) heated to $2000{ }^{\circ}$ C. Surface ionized potassium isotopes were then accelerated and mass separated by the high-resolution mass separator (HRS), before being cooled and bunched in the gas-filled linear Paul trap (ISCOOL) [45]. The ion bunches released from ISCOOL were 


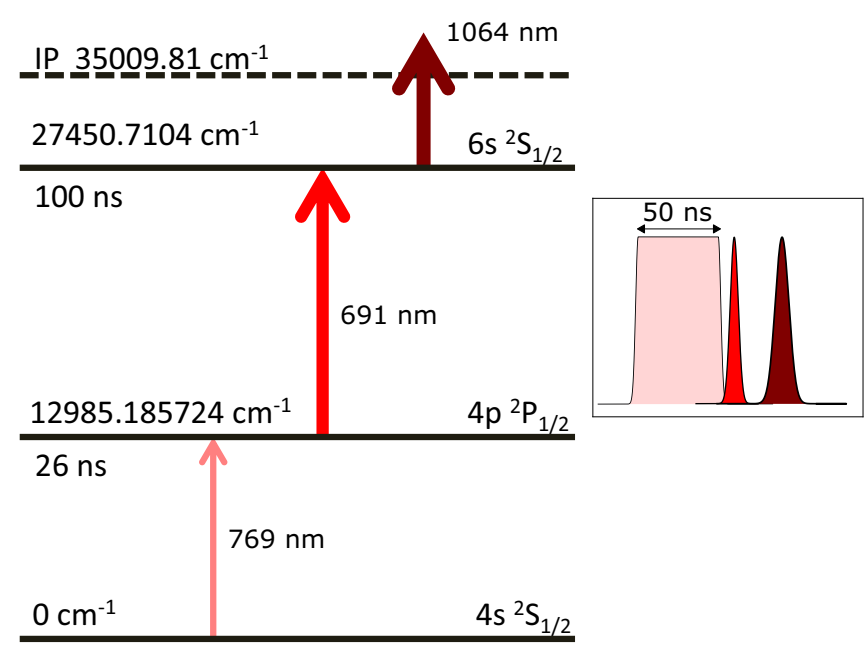

FIG. 1. Three-step ionization scheme used for the measurement of ${ }^{38-47} \mathrm{~K}$. After two resonant excitations the atoms are finally ionized by a nonresonant excitation of an electron from the $6 s^{2} S_{1 / 2}$ state to above the ionization potential (IP). The inset shows the sequence of laser pulses used for the high-resolution laser spectroscopy measurement.

accelerated to $40 \mathrm{keV}$ and guided towards the CRIS beam line [30], where the ions passed through a charge-exchange cell (the pressure of the chamber was under $7 \times 10^{-8} \mathrm{mbar}$ ) filled with potassium vapor at $160^{\circ} \mathrm{C}$. Around $70 \%$ of the in-flight ions were neutralized in this process. The neutralized fraction was estimated by comparing the current of the full beam (containing both the atoms and ions) measured on a Faraday cup (FC) after the charge-exchange cell to the current corresponding to the atomic beam. A bias voltage of a few tens of volts was applied to the outer ring of the FC to enhance the extraction of secondary electrons upon beam impact, hereby enabling the measurement of neutral beam currents. The pure atom beam was obtained by deflecting away the ions from the beam by applying high voltage on deflector plates placed before the FC. During the measurements of the hyperfine spectra of isotopes, the non-neutralized ions were always removed from the beam by using these electrostatic deflector plates. After passing through a differential pumping region, the atom bunch was overlapped with laser light in a $1.2 \mathrm{~m}$ long interaction region, which was held at pressure of $1.8 \times 10^{-9}$ mbar to reduce the collisional ionization.

The three-step resonance ionization scheme employed for the present experiment is shown in Fig. 1. The first excitation step is produced by a continuous wave (cw) titanium-sapphire (Ti:Sa) laser (M-Squared SolsTiS) pumped by a $18 \mathrm{~W}$ pump laser at $532 \mathrm{~nm}$ (Lighthouse Photonics). To prevent optical pumping to dark states, this laser light was chopped into $50 \mathrm{~ns}$ pulses at a repetition rate of $100 \mathrm{~Hz}$ by using a Pockels cell, as described in Ref. [36]. The wavelength of this laser was tuned to probe the hfs of the $4 \mathrm{~s}^{2} \mathrm{~S}_{1 / 2} \rightarrow 4 p^{2} \mathrm{P}_{1 / 2}$ transition at $769 \mathrm{~nm}$. Atoms in the excited $4 p^{2} \mathrm{P}_{1 / 2}$ state were then excited to the higher $6 s^{2} S_{1 / 2}$ state by a pulsed dye laser (Spectron PDL SL4000) with a spectral bandwidth of $10 \mathrm{GHz}$. This dye laser cavity was pumped by a frequency doubled Nd:YAG pulsed laser (Litron TRLi 250-100) at a repetition rate of
$100 \mathrm{~Hz}$. The fundamental light of the Litron laser (1064 nm) was used for the final nonresonant ionizing step. The ions created in the interaction region were separated from the nonionized atoms using deflector plates, and guided towards the microchannel plate (MCP Hamamatsu F4655-12). The arrival of ion bunches and laser pulses in the interaction region were synchronized and controlled using a Quantum Composers 9520 Series multichannel pulse generator [30]. The timing of each laser pulse could be adjusted independently, allowing the systematic investigation of the effect of different laser pulse sequences on the spectral line shape and extracted observables from experimental hfs spectra.

Figure 1 also shows an example of the time sequence of laser pulses optimized during the systematic survey of the effect of different laser timings on the hfs spectra, as will be discussed in Sec. III D. This time sequence of laser pulses provides us with the optimal balance between efficiency and resolution, and furthermore minimizes unwanted line shape distortions. It is therefore used for the evaluation of the precision and accuracy of the CRIS method in the $\mathrm{K}$ mass region, as presented in Secs. III B and III C.

In order to evaluate the applicability and precision of laser frequency tuning for high-resolution resonance ionization spectroscopy measurements of light atomic systems, additional diagnostics were introduced into the CRIS laser laboratory and a HighFinesse WS/U-2 wavelength meter. The key component of the monitoring system is a Toptica saturated absorption spectroscopy unit, which consists of a diode laser (DLPRO780), a temperature controlled vapor cell filled with $\mathrm{K}, \mathrm{Rb}$, and $\mathrm{Cs}(\mathrm{COSY})$ and the related locking electronics as shown in Fig. 2. In this experiment, the frequency of the diode laser was locked to the $F=2 \rightarrow F=3$ transition of the $D 1$ line in ${ }^{87} \mathrm{Rb}$, which is better resolved than the transitions in the $\mathrm{K}$ spectrum. The diode laser light was coupled into the high precision wavelength meter, as well as into a FabryPerot Interferometer (FPI: FPI-100-0750-1) with a $1 \mathrm{GHz}$ free spectral range and $5 \mathrm{MHz}$ typical resolution. This FPI cavity was locked to the diode laser frequency using TEM LaserLock digital locking electronics. A small fraction of cw laser beam from the Ti:Sa cavity was also sent into the wavelength meter and the FPI. The transmission fringes of the frequency scanned Ti:Sa laser through the locked FPI were monitored by using a Thorlabs DET10A/M-Si Detector and finally recorded by using a National Instruments USB-6211 acquisition card. In this configuration, the wavelength of both the diode and the Ti:Sa laser could be monitored and recorded throughout the duration of the entire experiment.

Since the frequency of the diode laser is locked to a well-known hyperfine transition of ${ }^{87} \mathrm{Rb}$ at $384228115.210(7) \mathrm{MHz}$, it provides a suitable frequency reference to monitor any frequency drifts during the experiment. Given the $11 \mathrm{~nm}$ difference between this laser light and the one used for the transition probed in the potassium isotopes, it was assumed that the response of optical elements inside of the wavelength meter and the FPI is the same for both laser lights. The monitoring was realized by continuously sampling the wavelength of the diode laser using the wavelength meter, and by recording the Ti:Sa transmission fringes of the locked FPI. 


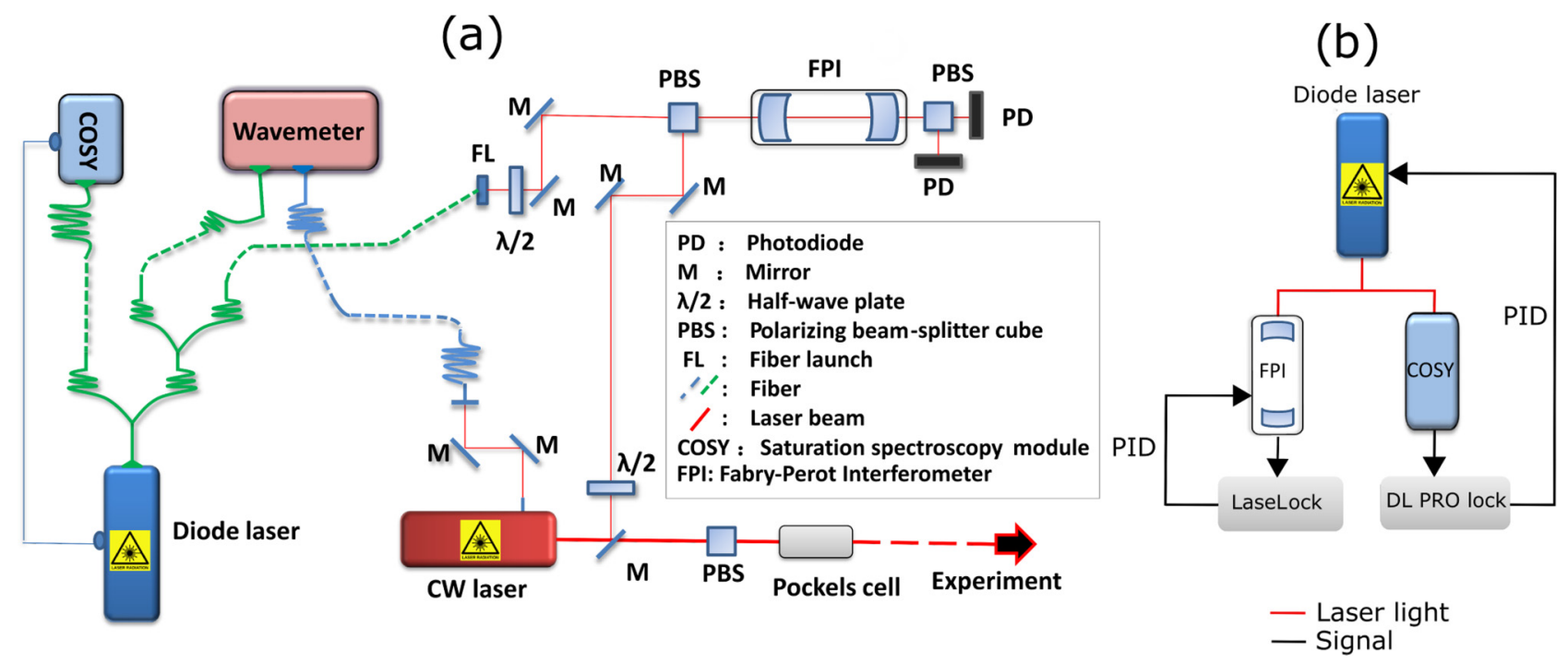

FIG. 2. Laser frequency stability monitoring system used for the experiment, see text for details.

\section{EXPERIMENTAL PROCEDURES, RESULTS, AND DISCUSSION}

The hfs of $38,39,42,44,46,47 \mathrm{~K}$ for the $4 s^{2} \mathrm{~S}_{1 / 2} \rightarrow 4 p^{2} \mathrm{P}_{1 / 2}$ atomic transition (Fig. 1) were measured with the CRIS technique. In this work, ${ }^{47} \mathrm{~K}$ was used as the reference isotope, the hfs of which was measured regularly during the experiment. The typical hfs spectrum of ${ }^{47} \mathrm{~K}$ is shown in Fig. 3(b). This ensures that slow drifts of the devices, such as wavelength meter and the acceleration voltage, are monitored.

The hfs $A$ parameters of the ground and first excited state $\left(A_{1}, A_{\mathrm{u}}\right.$, respectively) and the atomic field factor $F$ of the potassium isotopes are smaller $[11,41,42]$ than those in the middle- and heavy-mass regions previously measured with the CRIS technique [6,24,27,29]. Therefore, in order to extract reliable and high-precision experimental observables

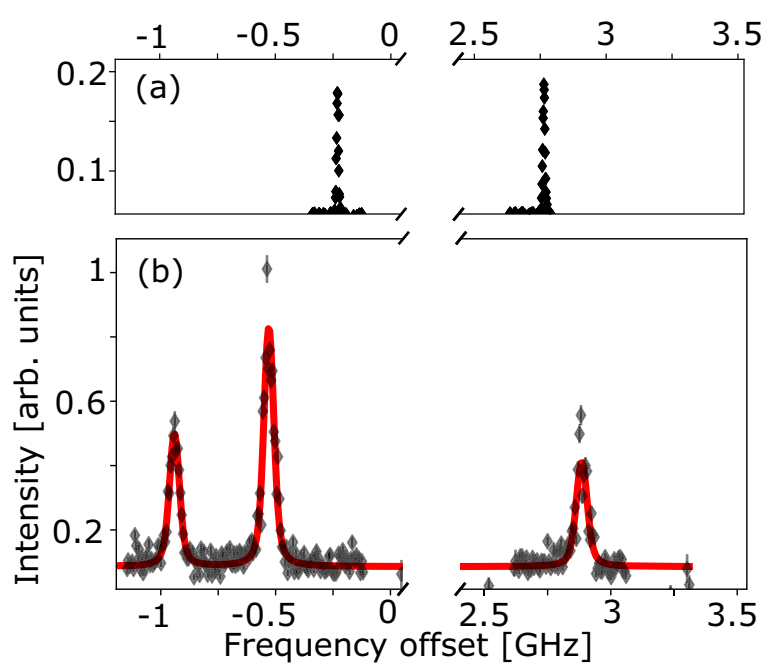

FIG. 3. (a) Transmission fringes through the FPI recorded on the photodiode as the function of the frequency of the scanning laser; (b) hfs spectrum of ${ }^{47} \mathrm{~K}$ taken at the same time. from the measured hfs spectra, it is crucial to monitor the drifts of various parameters, e.g., the frequency of the scanned Ti:Sa laser and the $40 \mathrm{keV}$ high voltage applied to ISCOOL (ISCOOL voltage), and to correct for those drifts as described in the following sections.

\section{A. Frequency scanning approach}

As mentioned above, there are two approaches to measure the hfs spectra of isotopes of interest in the collinear geometry: laser-frequency tuning or ion beam velocity tuning. Ion beam velocity tuning is performed by varying an accelerating or decelerating potential, while the laser frequency is locked to a fixed frequency $v_{\text {laser. }}$. Due to the Doppler effect, an ion or atom that is accelerated to an energy $V$ observes a shifted laser frequency $v$, given by

$$
\begin{gathered}
v=v_{\text {laser }} \sqrt{\frac{1-\beta}{1+\beta}}, \\
\text { where } \beta=\sqrt{1-\frac{m^{2} c^{4}}{\left(e V+m c^{2}\right)^{2}}} .
\end{gathered}
$$

This approach is commonly used for collinear laser spectroscopy setups worldwide $[9,46]$.

In the case of the CRIS technique, multiple laser steps are used to resonantly ionize atoms, which makes the voltage scanning more complex. To achieve high ionization efficiency, multiple laser beams are required to be perfectly overlapped with the atomic beam in the long interaction region of the CRIS setup. Tuning the ion beam velocity can affect significantly the focus and path of the atomic beam, and also potentially shift the frequency of the second excitation step off the resonance of the atoms, which will reduce the ionization efficiency. Furthermore, the voltage applied to the deflector plate in front of the ion detector has to change synchronously with the accelerating/decelerating potential used for Doppler 

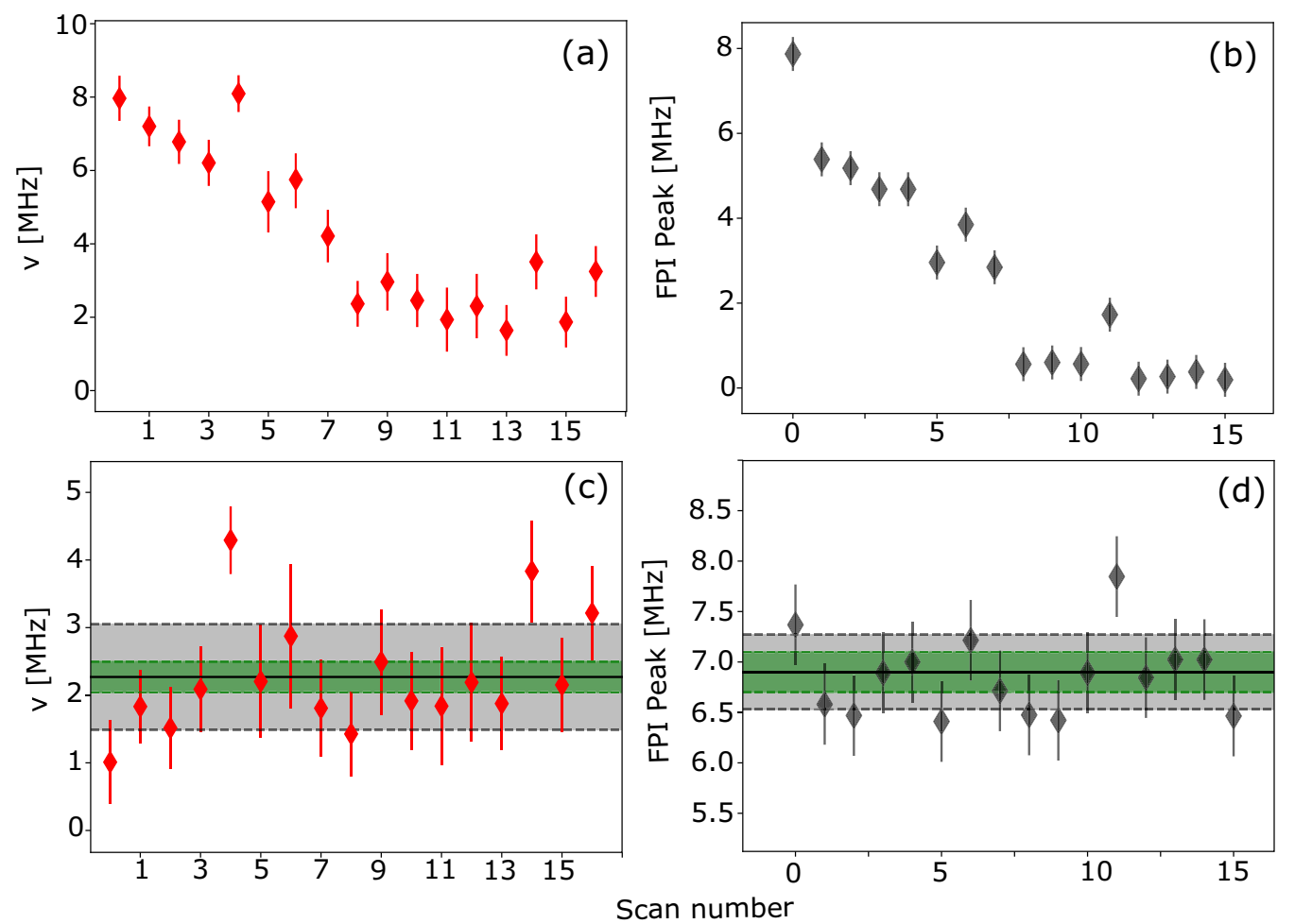

FIG. 4. The hfs COG extracted from repeated measurements of ${ }^{47} \mathrm{~K}$ (a) before and (c) after applying the correction described in the text. The position of the transmission fringes of the scanning laser in the locked FPI (b) before and (d) after applying the same frequency correction procedure. The frequency scale on $y$ axis is given relative to an offset.

tuning. Therefore, all measurements so far with CRIS have relied upon scanning the laser frequency.

\section{B. Evaluating the precision and accuracy}

Accurate and stable laser wavelength measurements are crucial for high-precision laser spectroscopy using the CRIS method. Many environmental factors can influence the frequency measured by the wavelength meter (e.g., the pressure and temperature of the laboratory that houses the wavelength meter). Therefore, changes or drifts in the range of 10-20 MHz from the wavelength meter reading over a long period of time have to be taken into account. In the following, an approach to correct these effects will be introduced in detail, and applied to the isotopes shift measurements of ${ }^{38-47} \mathrm{~K}$.

The frequency of the stabilized diode laser and the scanning Ti:Sa laser were recorded by the wavelength meter throughout the experiment. In order to extract the hfs parameter and center-of-gravity (COG) of the hyperfine spectrum in the rest frame of the atom, the laser frequencies recorded by the wavelength meter were Doppler shifted according to Eq. (3) using the event-by-event recorded ISCOOL voltage. In addition to this, the absolute voltage of ISCOOL was calibrated using results obtained by measurements on thermal beams [38]. Note that the same calibration was performed for the results presented in Ref. [11], with details on the calibration procedure given in Refs. [47,48]. The hfs spectra were fitted using the SATLAS package [49]. Figure 4(a) shows the $\mathrm{COG}$ of the reference isotope ${ }^{47} \mathrm{~K}$ during the experiment.
A clear drift of about $8 \mathrm{MHz}$ is observed, which comes mainly from a drift in the wavelength meter.

In order to remove this drift, the frequency of the Ti:Sa laser $\left(v^{i}\right)$ was calibrated for each recorded point $v_{\text {recorded }}^{i}$ by the following way:

$$
v^{i}=v_{\text {recorded }}^{i}-\delta v_{\text {diode }}^{i},
$$

where the $\delta v_{\text {diode }}^{i}=v_{\text {diode }}^{i}-v_{\text {diode }}^{0}$ indicates the change in the wavelength meter readout of the stabilized diode laser with respect to the $v_{\text {diode }}^{0}$. Note that this calibration procedure assumes that the wavelength meter drift for both Ti:Sa laser and diode laser is the same. After applying this calibration, a consistent value is obtained for the COG, as shown in Fig. 4(c). A standard deviation of $\sigma=0.77 \mathrm{MHz}$ is reached [gray wider band in Fig. 4(c)]. The error of the weighted mean $(0.23 \mathrm{MHz})$ is shown with the green narrow band.

To further evaluate the reliability of the calibration procedure and the achieved precision, and to verify if the frequency correction indeed accounts for wavelength meter drift, we take full advantage of the FPI system described in Fig. 2 and Sec. II. An example of two transmission fringes of the Ti:Sa laser from the FPI is shown in Fig. 3(a). Since the FPI cavity is locked to the stabilized diode laser, these transmission fringes should occur for the same laser frequency with a precision of less than $1 \mathrm{MHz}$ throughout the experiment only if the calibration procedure was performed correctly. Therefore, at least one FPI transmission fringe was recorded for each hfs measurement. Given the large hfs $A_{1}$ parameter of ${ }^{47} \mathrm{~K}$, the frequency scanning range was divided into two regions, as 
TABLE I. Comparison of the hyperfine $A_{\mathrm{l}}$ and $A_{\mathrm{u}}$ parameters measured in this work to the literature values taken from Refs. [50] and [38]. All hyperfine parameters are given in $\mathrm{MHz}$.

\begin{tabular}{|c|c|c|c|c|c|c|c|}
\hline$A$ & $I^{\pi}$ & $A_{1}$ & $A_{1}$ from $[50]$ & $A_{1}$ from $[38]$ & $A_{\mathrm{u}}$ & $A_{\mathrm{u}}$ from $[50]$ & $A_{\mathrm{u}}$ from $[38]$ \\
\hline 38 & $3^{+}$ & $404.2(5)$ & $404.3(3)$ & / & $49.0(6)$ & $48.9(2)$ & / \\
\hline 39 & $3 / 2^{+}$ & 231.1(3) & $231.0(3)$ & $231.0(3)$ & 27.1(6) & $27.8(2)$ & $27.5(4)$ \\
\hline 44 & $2^{-}$ & $-379.1(7)$ & $-378.9(4)$ & $-378.1(11)$ & $-45.7(3)$ & $-45.8(2)$ & $-44.9(11)$ \\
\hline 46 & $2^{-}$ & $-463.0(4)$ & $-462.8(3)$ & $-465.1(12)$ & $-55.4(3)$ & $-55.9(2)$ & $-55.7(13)$ \\
\hline 47 & $1 / 2^{+}$ & $3413.3(4)$ & $3413.2(3)$ & $3420.2(29)$ & $410.0(5)$ & $411.8(2)$ & $411.9(50)$ \\
\hline
\end{tabular}

shown in Fig. 3(b). In both low- and high-frequency range of the hfs spectrum, a transmission fringe from the FPI was recorded, as shown in Fig. 3(a).

Figure 4(b) shows the position of one FPI peak recorded during the experiment, before applying any frequency correction. A clear and significant drift of about $8 \mathrm{MHz}$ is observed, which closely matches the drift observed in the COG of ${ }^{47} \mathrm{~K}$ as shown in Fig. 4(a), and confirms that this drift is induced by the wavelength meter. The same point-by-point frequency correction was applied for the FPI transmission fringe. The FPI peak position after this frequency correction is presented in Fig. 4(d). A consistent value is achieved for all the measurements during the entire experiment. A standard deviation of $0.4 \mathrm{MHz}$ was obtained, as presented in Fig. 4(d) with a gray wider band, further supporting the precision achieved for the COG of ${ }^{47} \mathrm{~K}$ isotope demonstrated above. The $0.2 \mathrm{MHz}$ error of the weighted mean is shown on the same figure in green narrow band.

\section{Extracted hyperfine parameters, isotope shifts, and nuclear charge radii}

In order to evaluate the performance of CRIS technique and the frequency scanning method, it is necessary to compare the extracted hfs parameters and the isotope shift of each isotope to available literature values. After applying the aforementioned frequency correction (Sec. III B) to the hfs spectra of ${ }^{38-47} \mathrm{~K}$, the hfs parameters were extracted, as listed in Table I. They are in excellent agreement with the literature values and reach a similar precision as in Ref. [50].

From the deduced $\mathrm{COG}\left(v^{\mathrm{A}}\right)$ of the ${ }^{38-47} \mathrm{~K}$ isotopes $\delta v^{47, A}=v^{\mathrm{A}}-v^{47}$ were evaluated with respect to the COG $\left(v^{47}\right)$ of the reference isotope ${ }^{47} \mathrm{~K}$. These isotope shifts are presented in Table II and compared to those from the previous measurements, in which different experimental techniques have been used [11,38]. Since the CRIS technique and the collinear laser spectroscopy technique used in Ref. [38] are both applied to accelerated beams, the accuracy of the acceleration potential calibration has to be addressed in the error budget. The resulting systematic uncertainties of the isotope shifts are added in square brackets in Table II. These were propagated to obtain the systematic uncertainty of the $\delta\left\langle r^{2}\right\rangle$. Note that in Ref. [11], the systematic error of the $\delta\left\langle r^{2}\right\rangle$ was presented together with the large uncertainty related to the atomic field and mass factors. Our results for the isotope shift are in excellent agreement with the measurements from the atomic beam experiment [38]. They also agree with those from the optically detected collinear laser spectroscopy measurement [11], if the systematic error from those data is taken into account. Using the atomic mass shift factor $K_{\mathrm{MS}}=198.2(38) \mathrm{GHz}$ u and field shift factor of $F=$ -110 (3) $\mathrm{MHz} / \mathrm{fm}^{2}$, evaluated in Refs. [9,11], the changes in mean-square charge radii of ${ }^{38-47} \mathrm{~K}$ are deduced and presented in Table II and Fig. 5(a). The gray shaded area around the data points represents the large systematic error on the $\delta\left\langle r^{2}\right\rangle$, which results from the error on the atomic field and mass factors, as presented in the last column of Table II.

The agreement between the results presented in this work and literature values is further demonstrated in Fig. 5(b), where the deviation between our data, results from Refs. [11] and [38] is shown. The shaded bands indicate the systematic uncertainties related to the voltage calibration. The results provide confidence in the accuracy of the frequency calibration method and the frequency scanning approach. Furthermore they highlight the importance of the estimation of systematic uncertainties, which are often present when laser

TABLE II. The isotope shifts and changes in the mean-square charge radii obtained in this work relative to the reference isotope ${ }^{47} \mathrm{~K}$ are presented along with available results from literature. Systematic errors on the isotope shift and the resulting systematic uncertainties of the $\delta\left\langle r^{2}\right\rangle$ are added in the square brackets where available. The isotope shifts from Ref. [38] are recalculated using ${ }^{47} \mathrm{~K}$ as a reference. The last column shows the systematic uncertainty for $\delta\left\langle r^{2}\right\rangle$, coming from the error on the atomic $K_{\mathrm{MS}}, F$ factors, see text for more details. The isotope shifts are given in $\mathrm{MHz}$ and the $\delta\left\langle r^{2}\right\rangle$ in $\mathrm{fm}^{2}$.

\begin{tabular}{|c|c|c|c|c|c|c|c|}
\hline$A$ & $\mathrm{IS}_{1}$ from $[38]$ & $\mathrm{IS}_{2}$ from $[11]$ & IS $_{\text {CRIS }}$ & $\delta\left\langle r^{2}\right\rangle$ from $\mathrm{IS}_{1}$ & $\delta\left\langle r^{2}\right\rangle$ from $\mathrm{IS}_{2}$ & $\delta\left\langle r^{2}\right\rangle_{\mathrm{CRIS}}$ & $\sigma_{\mathrm{K}, \mathrm{F}}$ \\
\hline 38 & $-984.5(56)$ & $-985.9(4)[34]$ & $-983.8(4)[18]$ & $-0.14(5)$ & $-0.127(4)[31]$ & $-0.146(4)[16]$ & 0.17 \\
\hline 39 & $-857.5(17)$ & $-862.5(9)[30]$ & $-858.4(6)[5]$ & $-0.080(15)$ & $-0.037(8)[27]$ & $-0.074(5)[5]$ & 0.15 \\
\hline 44 & $-293.2(22)$ & $-292.1(5)[10]$ & $-293.19(56)[23]$ & $0.047(20)$ & $0.037(5)[9]$ & $0.0464(51)[21]$ & 0.05 \\
\hline 46 & $-94.7(23)$ & $-91.6(5)[3]$ & $-95.81(55)[6]$ & $0.026(21)$ & $-0.002(5)[3]$ & $0.0363(50)[5]$ & 0.01 \\
\hline
\end{tabular}




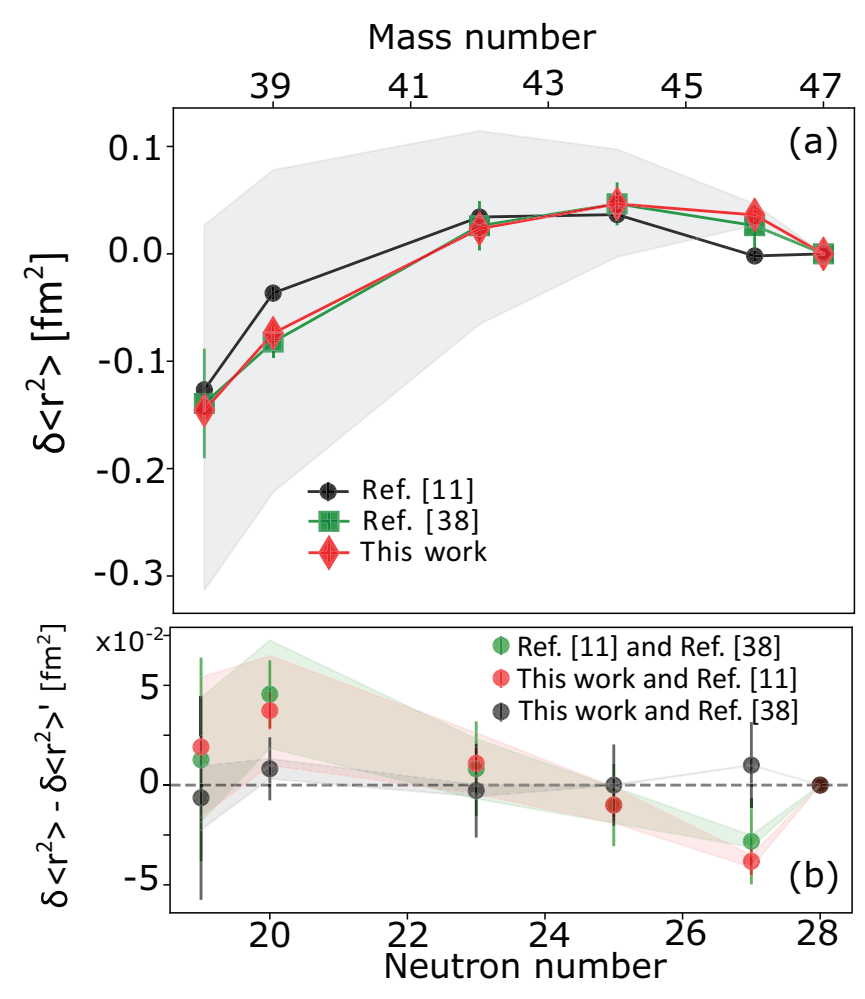

FIG. 5. (a) Changes in the mean-square charge radii, $\delta\left\langle r^{2}\right\rangle$, obtained in this work, are compared to those from literature $[11,38]$. The gray band represents the systematic uncertainty from atomic $K_{\mathrm{MS}}$ and the $F$. (b) The deviations between $\delta\left\langle r^{2}\right\rangle$ values reported in Table II are presented taking into account the systematic uncertainties due to the voltage calibration (shaded areas).

spectroscopy is performed on accelerated beams. However, they only become significant when an isotopic chain of light mass elements is studied.

\section{Systematic investigation of line shapes}

The spectral line shape in the hfs spectra observed in a collinear laser spectroscopy measurement is usually assumed to be a Voigt profile, which is the convolution of a Lorentzian and a Gaussian line shape. The Lorentzian component is a homogeneous line broadening due to the finite lifetime of the atomic states and the laser power [51,52], while the Gaussian part is associated with inhomogeneous broadening mechanisms such as the energy spread of the ion beam. The line shape of hfs spectra obtained with the CRIS technique is sometimes further modified due to the interaction between the atoms and the electromagnetic field of the pulsed lasers. During the charge exchange process, excitations and deexcitations due to collisions with the alkali atoms often happen when the CEC is operated at higher temperature, leading to asymmetric spectral line shapes, and appearance of side peaks [53]. The latter has not been observed during the measurement presented in this work. An AC Stark effect on the other hand, caused by the high power of the pulsed lasers, can cause another undesired asymmetric line shape distortion [35,43,54], which will be discussed in Sec. III D 1.
Delayed ionization Simultaneous excitation Simultaneous ionization

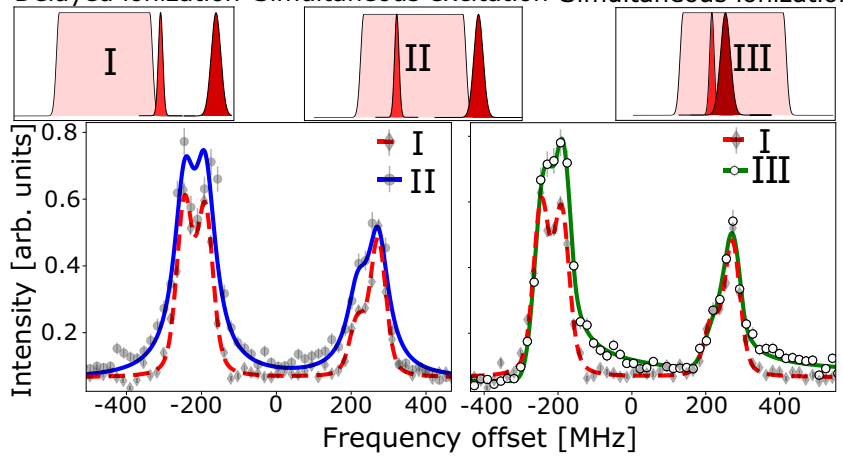

FIG. 6. The line shape of hfs peaks in spectra of ${ }^{39} \mathrm{~K}$ observed using delayed ionization (I: dashed red line and gray diamonds), simultaneous excitation (II: solid blue line and full circles) and simultaneous ionization (III: solid green line and open circles), as explained in the text.

Although there are many studies on laser-induced line shape effects $[36,43,55]$, the complexity of the three-step laser ionization scheme used in this work makes it challenging to derive an analytical expression for the final line shape [43]. As observed in our earlier work [29], the experimental hfs spectra obtained with three-step laser ionization are influenced by an AC Stark effect, which can be approximated with an asymmetric line shape function.

In this work, the hfs spectra obtained with $90 \mathrm{~mW}$ laser power in the first excitation step and laser pulse structure shown in Fig. 1 optimized for high resolution, were analyzed using a Voigt-profile. For other cases in which the line shape was strongly influenced by the laser power and time structure of pulsed lasers, the hfs spectra are found to match the asymmetric fitting profile defined by an asymmetric Gaussian function better than an asymmetric Lorentzian spectral line shape. All above-mentioned line shapes are implemented in the data analysis package SATLAS [49].

\section{Delayed ionization}

The delayed ionization method has been investigated with the CRIS technique [43], showing significant advantages for reaching high resolution and high efficiency. By ensuring the laser pulse used for resonance excitation has no temporal overlap with the ionizing laser pulse, line shape distortion from AC Stark shift can be avoided. It was also demonstrated in the earlier studies of $\mathrm{Cu}$ and Fr isotopes [43] that this delayed laser pulse does not sacrifice the overall ionization efficiency if a sufficiently long-lived excited state is used.

TABLE III. Sequence of laser pulses for different timing configurations shown in Fig. 6. The delays are calculated from the rising edge of the first laser pulse.

\begin{tabular}{lcccc}
\hline \hline Mode & 2nd delay & 3rd delay & FWHM [MHz] & Relative efficiency \\
\hline I & $65(5) \mathrm{ns}$ & $115(5) \mathrm{ns}$ & $47.8(12)$ & 0.33 \\
II & 28(5) ns & 60(5) ns & $69.3(18)$ & 0.86 \\
III & 28(5) ns & 35(5) ns & $53(2)$ & 1 \\
\hline \hline
\end{tabular}


TABLE IV. Relevant information for each laser step in the resonance ionization scheme.

\begin{tabular}{lcccc}
\hline \hline Step & Wavelength & Energy & Pulse length & Beam diameter \\
\hline 1st & $769 \mathrm{~nm}$ & $0.02 \mu \mathrm{J}^{\mathrm{a}}$ & $50 \mathrm{~ns}$ & $0.8 \mathrm{~cm}$ \\
2nd & $691 \mathrm{~nm}$ & $300 \mu \mathrm{J}$ & $10 \mathrm{~ns}$ & $1 \mathrm{~cm}$ \\
3rd & $1064 \mathrm{~nm}$ & $75 \mathrm{~mJ}$ & $20 \mathrm{~ns}$ & $1 \mathrm{~cm}$ \\
\hline
\end{tabular}

$365 \mathrm{~mW}$ of cw light chopped into $50 \mathrm{~ns}$ pulses.

In the case of potassium, the lifetime of the first excited state $(26 \mathrm{~ns})$ is shorter than that used for the studies of francium and copper atoms, having lifetimes of $83 \mathrm{~ns}$ and $358 \mathrm{~ns}$ respectively. The use of a Pockels cell for chopping the $\mathrm{cw}$ laser light, resulted in $50 \mathrm{~ns}$ minimal pulse lengths of the first step, almost twice as long as the lifetime of the excited state. This introduced some degree of optical pumping, which acts to reduce the total ionization efficiency. Furthermore, total temporal separation of the laser pulses will result in a drop in efficiency due to spontaneous decay of the first excited state.

The spectral line shape of the of ${ }^{39} \mathrm{~K}$ hyperfine transition peaks was systematically investigated under three different timing configurations of the laser pulses, referred to as delayed ionization (I), simultaneous excitation (II), and simultaneous ionization (III) as shown in the top panel of Fig. 6 . In Table III, the delays of the second and third laser steps are summarized for these three configurations. Information on each laser step (power, pulse length, beam size) is also summarized in Table IV.

The achieved hyperfine spectra under these three configurations are presented in Fig. 6, with a normalized $y$ axis for an easier comparison of line shapes. The full width at half maximum (FWHM) and relative efficiency extracted from these hfs spectra are also summarized in Table III. An unperturbed $\mathrm{hfs}$ spectrum is obtained using delayed ionization, as shown with a dashed red fitted line in Fig. 6, with the FWHM of less than $50 \mathrm{MHz}$. This timing configuration of laser pulses was used for the measurements discussed previously in Sec. III B and Sec. III C.

In the case of simultaneous excitation (see Fig. 6 and Table III), the pulse of the second laser arrives $37 \mathrm{~ns}$ earlier than in the case of delayed ionization, and overlaps with the first laser pulse, while the third laser used for ionization is still decoupled from the first two laser pulses. This configuration results in a broadened [FWHM: 69.3(18) MHz] but symmetric Voigt line shape, which was fitted with the solid blue line in Fig. 6 left. An intuitive interpretation is that the presence of the second laser decreases the effective lifetime of the excited state, resulting in a broadened resonance. The spectrum fitted with the solid green line in Fig. 6 right, is obtained with all three laser pulses overlapped (simultaneous ionization). The highest efficiency is reached under this condition, but the line shape features a long $2 \mathrm{GHz}$ tail on the high-frequency side. The absence of the asymmetric line shape with simultaneous excitation, comparing with the significant asymmetric spectrum observed with simultaneous ionization, indicates that the asymmetry is induced by the third, high-power ionization laser. This was observed also in earlier CRIS experiments $[29,36,43]$.

In order to gain a better understanding of the ionization efficiency losses, line shape distortions, and the effect these distortions may have on the extracted hyperfine parameters, laser resonance ionization spectroscopy was performed on ${ }^{39,42,47} \mathrm{~K}$ under the conditions of delayed and simultaneous
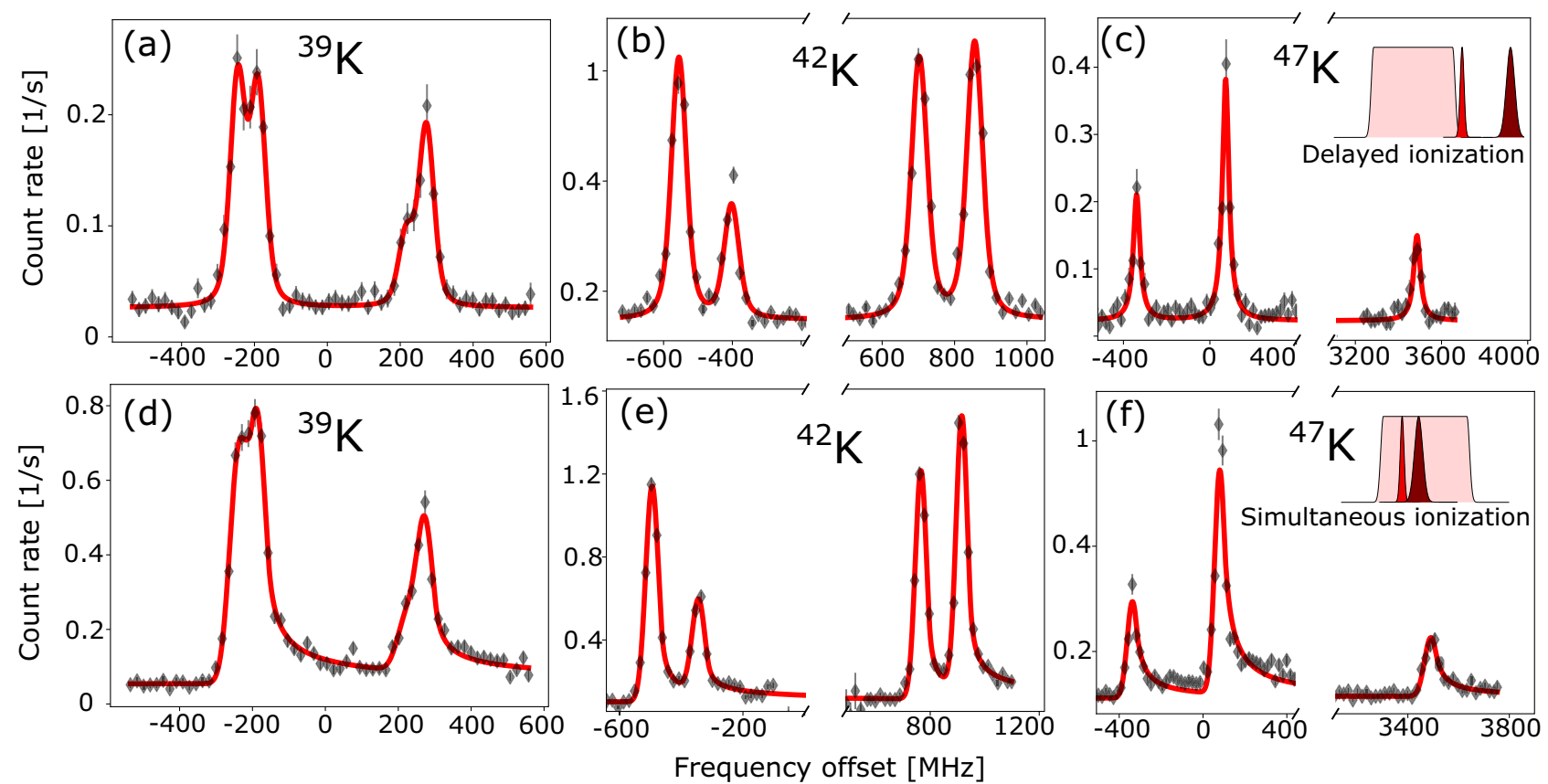

FIG. 7. Typical hfs spectra of ${ }^{39,42,47} \mathrm{~K}$ obtained using delayed [(a), (b), (c)] and simultaneous [(d), (e), (f)] ionization with $90 \mathrm{~mW}$ laser power of the first excitation laser. The time structures of the three laser pulses for these configurations are depicted in the hfs spectra of ${ }^{47} \mathrm{~K}$, (c) and (d). 

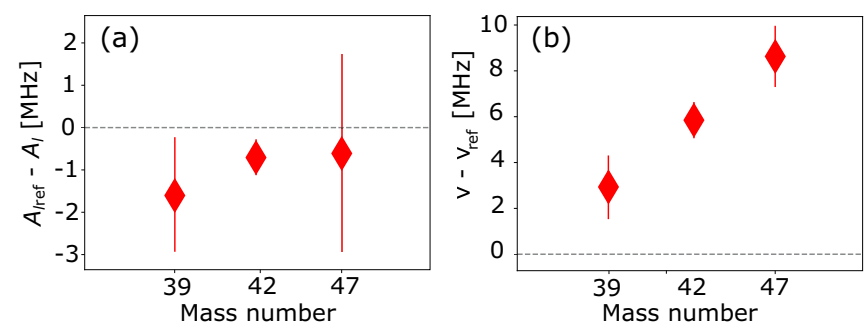

FIG. 8. Difference between the observables obtained from delayed and simultaneous ionization measurements for different isotopes ${ }^{39,42,47} \mathrm{~K}$. The observables are extracted from spectra shown in Fig. 7.

ionization. These three isotopes have different nuclear spins and hyperfine splittings. It therefore allows us to investigate if the differences in hyperfine splittings play a role in how the line shapes are distorted. Furthermore, as mentioned in Sec. III B, precise isotope shift measurements are of critical importance for light isotopes in order to precisely extract information on the nuclear size. Indeed, if the line shape distortion in the hfs spectra is different for each isotope, this will affect the accuracy of the isotope shift. Typical hfs spectra of ${ }^{39,42,47} \mathrm{~K}$ observed with delayed and simultaneous ionization are presented in the top and bottom panels of Fig. 7, respectively. The time structures of the arriving laser pulses are also presented schematically in the inset of Fig. 7(c) and $7(f)$. Significant changes in the line shape were observed for each isotope when overlapping the laser pulses, as well as an increase in the overall efficiency [Figs. 7(d), 7(e) and 7(f)].

Figure 8 plots the difference of hfs $A_{1}$ and COG (v) extracted from the spectra shown in Fig. 7 observed with simultaneous and delayed ionization. The presented results demonstrate that the simultaneous ionization condition, with all laser pulses overlapped, has a strong effect on the resulting COG [Fig. 8(b)] but less on the $A_{1}$ parameter [Fig. 8(a)]. Furthermore, the magnitude of the COG shift is different for each isotope. As a result, the extracted $\delta\left\langle r^{2}\right\rangle$ values are significantly larger than the values reported in Sec. III B as presented in Fig. 9. Since the isotope shift is calculated relative to the COG of ${ }^{47} \mathrm{~K}$, the $\delta\left\langle r^{2}\right\rangle$ of ${ }^{47} \mathrm{~K}$ is zero in both cases. As expected the 8.6(13) MHz difference observed for the COG of the reference isotope in these two configurations results in a significant change in the value of charge radii

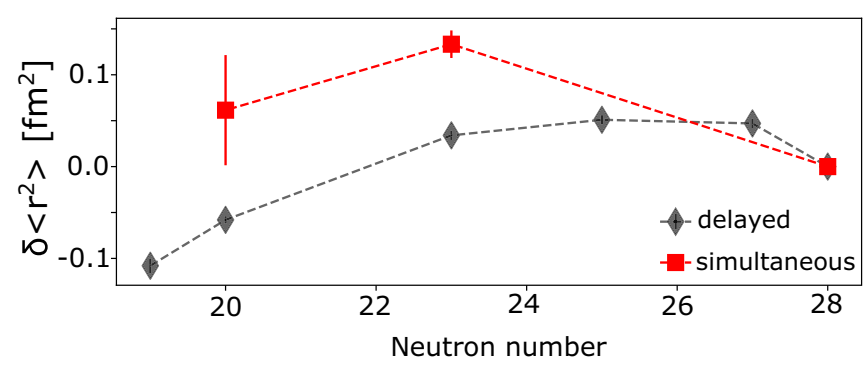

FIG. 9. The $\delta\left\langle r^{2}\right\rangle$ extracted with simultaneous ionization compared with $\delta\left\langle r^{2}\right\rangle$ measured with delayed ionization, as also plotted in Fig. 7.

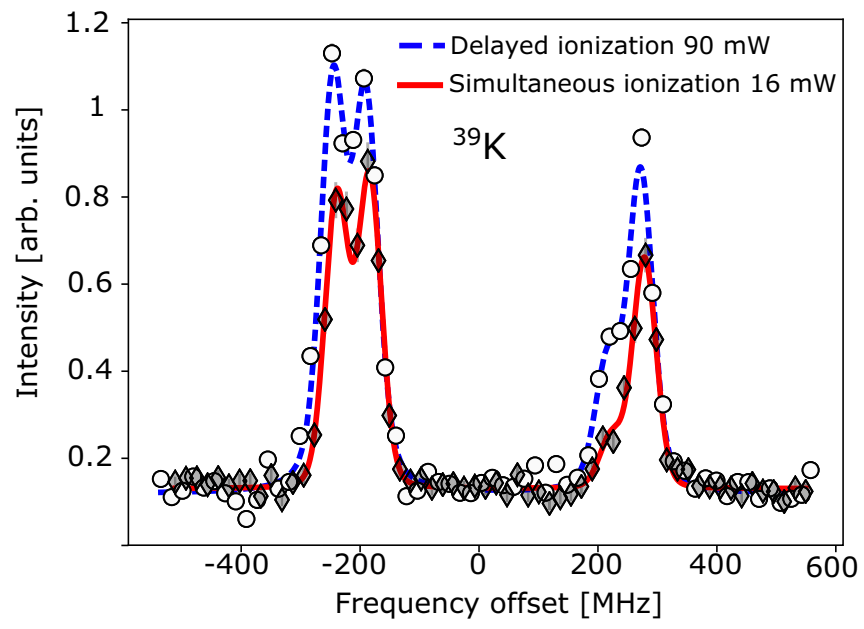

FIG. 10. Hyperfine spectrum of ${ }^{39} \mathrm{~K}$ taken with simultaneous ionization and $16 \mathrm{~mW}$ of laser power (solid red line) and the same structure obtained using delayed ionization and $90 \mathrm{~mW}$ laser power (dashed blue line). The count rates were normalized for better visualization. More details about the spectra can be found in Table V.

(Fig. 9). Thus, in order to extract nuclear parameters with a good precision and accuracy, simultaneous ionization with overlapped laser pulses should be always avoided, unless the above observed effects are well understood and controlled.

\section{Role of laser power in simultaneous ionization}

Using the configuration of simultaneous ionization, we measured the hfs spectra of ${ }^{39,42,47} \mathrm{~K}$ with four different laser powers for the first excitation step: $16 \mathrm{~mW}, 40 \mathrm{~mW}, 90 \mathrm{~mW}$, and $365 \mathrm{~mW}$. Figure 10 gives an example of the hfs spectrum of ${ }^{39} \mathrm{~K}$ (fitted with solid red line) taken with a laser power of $16 \mathrm{~mW}$ and simultaneous ionization. A high-resolution hfs spectrum was observed without obvious power broadening or evidence for AC Stark effect, even though the laser power of the second and third laser steps remained the same. An interpretation of this is not straightforward, but this measurement does illustrate the complexity of three-step laser ionization with high-power pulsed lasers. For comparison, the spectrum of ${ }^{39} \mathrm{~K}$ taken with delayed ionization and $90 \mathrm{~mW}$ laser power of the first resonant excitation is shown and fitted with dashed blue line in Fig. 10. The relevant information for laser power of first resonant excitation, the sequence of laser pulses and the achieved FWHM for the hfs spectra of ${ }^{39} \mathrm{~K}$ in Fig. 10 are summarized in Table V.

Figure 11 illustrates the difference of the hfs parameter $\left(A_{1}\right)$ and COG $(v)$ extracted from the hfs spectra of ${ }^{39,42,47} \mathrm{~K}$

TABLE V. Sequence of laser pulses for resonance ionization and the FWHM of hyperfine peaks for the spectra presented on Fig. 10 and Fig. 12.

\begin{tabular}{lcccc}
\hline \hline Isotope & Power [mW] & 2nd delay & 3rd delay & FWHM [MHz] \\
\hline${ }^{39} \mathrm{~K}$ & 16 & $28(5) \mathrm{ns}$ & $35(5) \mathrm{ns}$ & $46.9(16)$ \\
${ }^{39} \mathrm{~K}$ & 90 & $65(5) \mathrm{ns}$ & $115(5) \mathrm{ns}$ & $47.8(12)$ \\
${ }^{47} \mathrm{~K}$ & 365 & $20(5) \mathrm{ns}$ & $20(5) \mathrm{ns}$ & $105(5)$ \\
\hline \hline
\end{tabular}



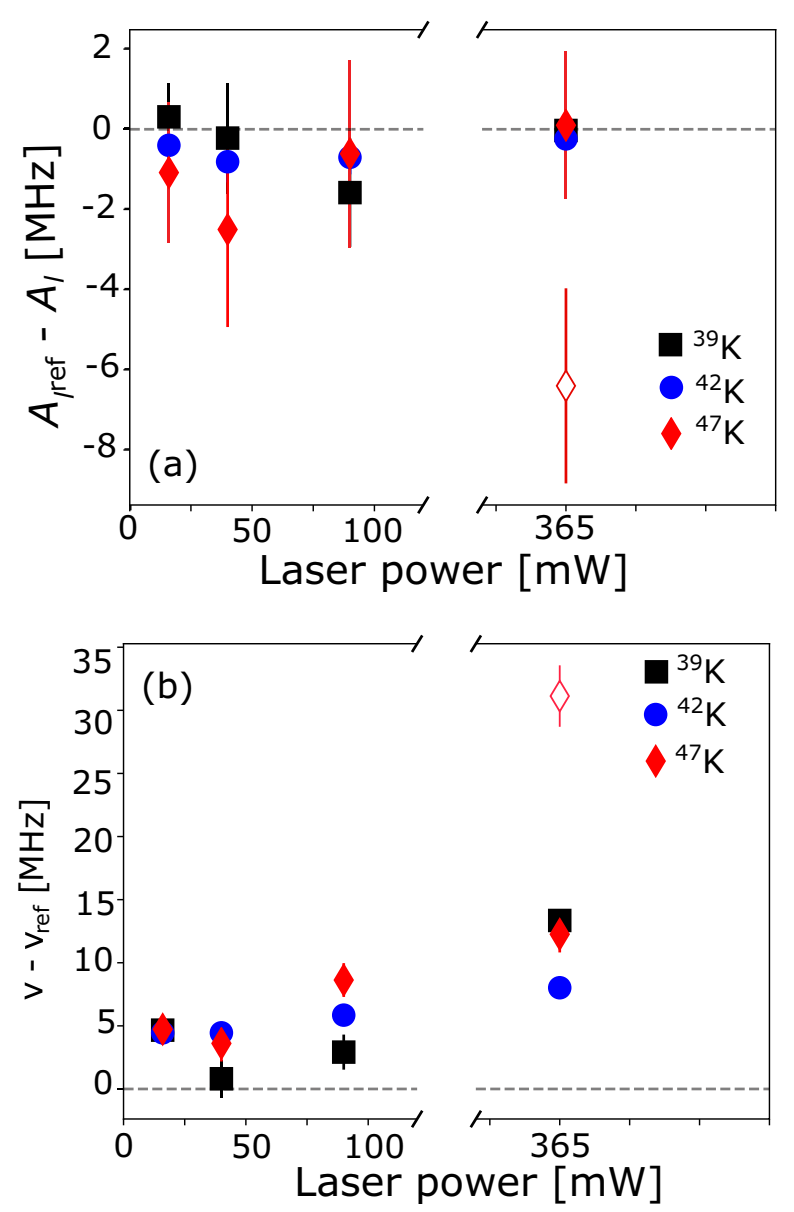

FIG. 11. Difference between (a) $A_{1}$ and hfs COG (v) (b) for ${ }^{39,42,47} \mathrm{~K}$ measured with simultaneous ionization for different laser powers. The data point marked with open diamond is extracted from the hfs spectrum shown in Fig. 12.

obtained from simultaneous ionization and delayed ionization, with different laser powers for the first excitation step (with the solid markers). There is no significant change observed for $A_{1}$ parameter [see Fig. 11(a)], although the broadened and asymmetric hfs spectra measured with higher power and overlapped laser pulses prevents resolving the individual hyperfine components of ${ }^{39} \mathrm{~K}$. However, the COG is clearly affected by the laser power, as an obvious increasing shift is observed when the laser power is increased, as shown in Fig. 11(b). The COG shift due to the presence of a high-power laser has been observed in the past [35]. Note that, in the case of lowest laser power $(16 \mathrm{~mW})$, the COG frequency observed with simultaneous ionization still deviates by about $5 \mathrm{MHz}$ [see Fig. 11(b)], even though a fairly high-resolution spectrum is observed (Fig. 10). This again underlines the importance of avoiding overlapped laser pulses in high-precision resonance ionization studies.

To further investigate the effect of laser pulse timings and powers on $A_{1}$ and $v$, the hfs spectrum of ${ }^{47} \mathrm{~K}$ was measured with the highest laser power $(365 \mathrm{~mW})$ and timing configuration shown in Fig. 12. In this case, the laser pulses of the second and third steps are overlapped just after the rising

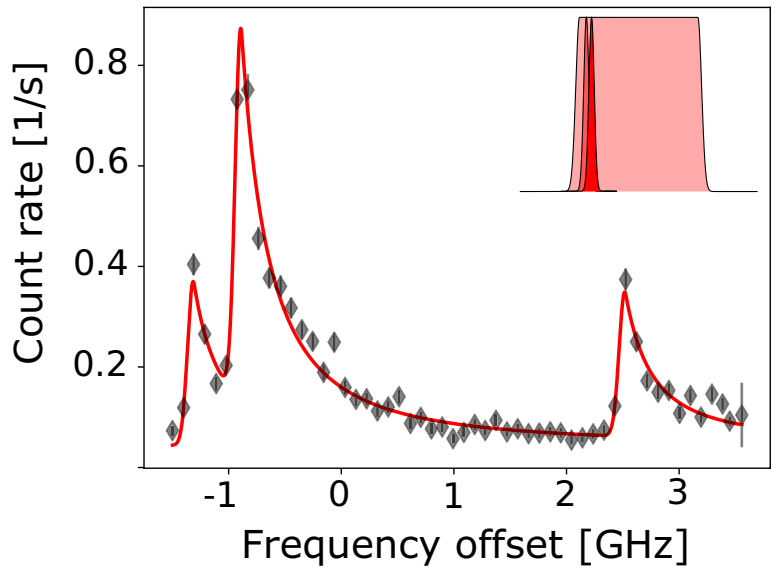

FIG. 12. Hyperfine spectrum of ${ }^{47} \mathrm{~K}$ taken with simultaneous ionization. The second and third step laser pulses are close to the rising edge of the first laser pulse and $365 \mathrm{~mW}$ of laser power is used for the first excitation step. More details about the spectrum can be found in Table V.

edge of the first laser pulse, resulting in the qualitatively worst line shape and broadening. The extracted $A_{1}$ and $v$ are also presented in Figs. 11(a) and 11(b), respectively, with the open red diamonds. Even for the $A_{1}$ parameter [Fig. 11(a)], although with a large error bar, a clear deviation of about $A_{\mathrm{I}_{\mathrm{ref}}}-A_{1}=7.3(24) \mathrm{MHz}$ is observed. This deviation is further enhanced for the extracted COG frequency, as plotted in Fig. 11(b), with a maximum deviation of 31.1 (24) $\mathrm{MHz}$ $\left(\delta v=v-v^{\text {ref }}\right)$.

\section{SUMMARY AND OUTLOOK}

In this paper, we presented a series of collinear resonance ionization spectroscopy measurements of the ${ }^{38,39,42,44,46,47} \mathrm{~K}$ isotopes with the CRIS setup. A precision and accuracy of less than $1 \mathrm{MHz}$ was reached for the hyperfine parameters, as well as for the isotope shifts thanks to the newly installed laser-frequency calibration and monitoring system. The extracted changes in mean-square charge radii are in a good agreement with those obtained with previous high-precision laser spectroscopy and atomic beam measurements when the systematic uncertainties related to beam acceleration are taken into account.

The line shapes of the hfs spectra of potassium isotopes observed with the three-step resonance ionization process were investigated with different laser powers and pulse timings. It was shown that narrow symmetric line shapes leading to precise and accurate results are obtained only if a specific time sequence between the three laser steps is applied. It was also confirmed that overlapping the laser pulses has a significant effect on the line shape, with clear broadening and asymmetries. This line shape distortion results in shifts of the hfs COG of several $\mathrm{MHz}$, and thus leads to inaccurate values for the deduced isotope shifts and charge radii. No significant deviations were observed for the extracted hyperfine $A_{1}$ parameter of the atomic $4 s^{2} S_{1 / 2}$ state, implying that the nuclear magnetic moments can still be reliably extracted 
in this case. Results of this work open the way for studies on lighter isotopes in the $Z=20$ region using the collinear resonance ionization techniques.

\section{ACKNOWLEDGMENTS}

We acknowledge the support of the ISOLDE collaboration and technical teams. This work was supported in part by the National Key R\&D Program of China
(Contract No. 2018YFA0404403), the National Natural Science Foundation of China (Grant No. 11875073); the BriX Research Program No. P7/12, FWO-Vlaanderen (Belgium), GOA 15/010 from KU Leuven; ERC Consolidator Grant No. 648381 (FNPMLS); the STFC consolidated Grants No. ST/L005794/1 and No. ST/L005786/1 and Ernest Rutherford Grant No. ST/L002868/1; the EU Horizon 2020 research and innovation programme through ENSAR2 (Grant No. 654002). We acknowledge the financial aid of the Ed Schneiderman Fund at New York University.
[1] B. Cheal and K. T. Flanagan, J. Phys. G: Nucl. Part. Phys. 37, 113101 (2010).

[2] P. Campbell et al., Prog. Part. Nucl. Phys. 86, 127 (2016).

[3] W. Nörtershäuser et al., Phys. Rev. Lett. 102, 062503 (2009).

[4] R. F. Garcia Ruiz et al., Nature Phys. 12, 594 (2016).

[5] X. F. Yang et al., Phys. Rev. Lett. 116, 182502 (2016).

[6] R. P. de Groote et al., Phys. Rev. C 96, 041302(R) (2017).

[7] M. Laatiaoui et al., Nature (London). 538, 495 (2016).

[8] R. Ferrer et al., Nature Commun. 8, 14520 (2017).

[9] K. Minamisono et al., Phys. Rev. Lett. 117, 252501 (2016).

[10] B. A. Marsh et al., Nature Phys. 14, 1163 (2018).

[11] K. Kreim et al., Phys. Lett. B 731, 97 (2014).

[12] A. J. Miller et al., Nature Phys. 15, 432 (2019).

[13] M. Hammen et al., Phys. Rev. Lett. 121, 102501 (2018).

[14] S. Rothe et al., Nature Commun. 4, 1835 (2013).

[15] M. R. Kalita et al., Phys. Rev. A 97, 042507 (2018).

[16] Z.-T. Lu, P. Mueller, G. W. F. Drake, W. Nortershauser, S. C. Pieper, and Z. C. Yan, Rev. Mod. Phys. 85, 1383 (2013).

[17] V. N. Fedosseev, Yu. Kudryavtsev, and V. I. Mishin, Phys. Scr. 85, 058104 (2012).

[18] S. L. Kaufman, Opt. Commun. 17, 309 (1976).

[19] R. Neugart, Hyperfine Interact. 24, 159 (1985).

[20] W. H. Wing et al., Phys. Rev. Lett. 36, 1488 (1976).

[21] H. De Witte et al., Phys. Rev. Lett. 98, 112502 (2007).

[22] T. E. Cocolios et al., Phys. Rev. Lett. 106, 052503 (2011).

[23] R. Ferrer et al., Phys. Lett. B 728, 191 (2014).

[24] K. T. Flanagan et al., Phys. Rev. Lett. 111, 212501 (2013).

[25] R. Heinke et al., Hyperfine Interact. 238, 6 (2016).

[26] T. E. Cocolios et al., Nucl. Instrum. Meth. B 317, 565 (2013).

[27] K. M. Lynch et al., Phys. Rev. C 97, 024309 (2018).

[28] S. G. Wilkins et al., Phys. Rev. C 96, 034317 (2017).

[29] G. J. Farooq-Smith et al., Phys. Rev. C 96, 044324 (2017).

[30] K. M. Lynch et al., Phys. Rev. X 4, 011055 (2014).

[31] H. Heylen et al., Phys. Rev. C 94, 054321 (2016).

[32] M. L. Bissell et al., Phys. Rev. C 93, 064318 (2016).
[33] L. Xie et al., Phys. Lett. B 797, 134805 (2019).

[34] W. H. King, Isotope Shifts in Atomic Spectra (Springer, Berlin, 1984).

[35] J. Bakos, Phys. Rep. 31, 209 (1977).

[36] R. P. de Groote et al., Phys. Rev. Lett. 115, 132501 (2015).

[37] W. Nörtershäuser et al., Phys. Rev. A 83, 012516 (2011).

[38] F. Touchard et al., Phys. Lett. B 108, 169 (1982).

[39] G. Huber et al., Phys. Rev. C 18, 2342 (1978).

[40] J. A. Behr, A. Gorelov, T. Swanson, O. Hausser, K. P. Jackson, M. Trinczek, U. Giesen, J. M. D’Auria, R. Hardy, T. Wilson, P. Choboter, F. Leblond, L. Buchmann, M. Dombsky, C. D. P. Levy, G. Roy, B. A. Brown, and J. Dilling, Phys. Rev. Lett. 79, 375 (1997).

[41] J. Papuga et al., Phys. Rev. Lett. 110, 172503 (2013).

[42] D. M. Rossi et al., Phys. Rev. C 92, 014305 (2015).

[43] R. P. de Groote, M. Verlinde, V. Sonnenschein, K. T. Flanagan, I. Moore, and G. Neyens, Phys. Rev. A 95, 032502 (2017).

[44] E. Kugler, Hyperfine Interact. 129, 23 (2000).

[45] E. Mané et al., Eur. Phys. J. A 42, 503 (2009).

[46] R. Neugart et al., J. Phys. G: Nucl. Part. Phys. 44, 064002 (2017).

[47] J. Papuga, Structure of potassium isotopes studied with collinear laser spectroscopy, Ph.D. thesis, KU LEUVEN, 2015.

[48] K. Kreim, Collinear laser spectroscopy of potassium: Nuclear charge radii beyond $\mathrm{N}=28, \mathrm{Ph} . \mathrm{D}$. thesis, Heidelberg University, 2013.

[49] W. Gins et al., Comput. Phys. Commun. 222, 286 (2018).

[50] J. Papuga et al., Phys. Rev. C 90, 034321 (2014).

[51] M. L. Citron, H. R. Gray, C. W. Gabel, and C. R. Stroud, Phys. Rev. A 16, 1507 (1977).

[52] N. Vitanov et al., Opt. Commun. 199, 117 (2001).

[53] N. Bendali et al., J. Phys. B: At. Mol. Phys. 19, 233 (1986).

[54] A. Kastler, J. Opt. Soc. Am. 53, 902 (1963).

[55] L. Li, B.-X. Yang, and P. M. Johnson, J. Opt. Soc. Am. B 2, 748 (1985) 